\title{
Tranexamic Acid-Induced Acute Renal Cortical Necrosis in Post-Endoscopic Papillectomy Bleeding
}

\author{
Doo Hyun Ko, Tae Hyung Kim, Jong Wook Kim, Ja Joong Gu, Baek Hyun Yoon, Ji Hong Oh and Seung Goun Hong \\ Department of Internal Medicine, SAM Anyang Hospital, Anyang, Korea
}

Acute renal failure can be the result of acute renal cortical necrosis (RCN), which commonly occurs from complications occurring during pregnancy. $\mathrm{RCN}$ is rarely caused by medications, although tranexamic acid, which is used in patients with acute bleeding for its antifibrinolytic effects, reportedly causes acute RCN in rare cases. An 82-year-old woman experienced gastrointestinal bleeding after endoscopic papillectomy of an ampullary adenoma. The bleeding was controlled with tranexamic acid administration; however, 4 days later, her urine volume decreased and she developed pulmonary edema and dyspnea. Serum creatinine levels increased from 0.8 to 3.9 $\mathrm{mg} / \mathrm{dL}$ and dialysis was performed. Abdominal pelvic computed tomography with contrast enhancement revealed bilateral RCN with no renal cortex enhancement. Renal dysfunction and oliguria persisted and hemodialysis was continued. Clinicians must be aware that acute RCN can occur after tranexamic acid administration to control bleeding. Clin Endosc 2017;50:609-613

Key Words: Kidney cortex necrosis; Tranexamic acid; Hemorrhage; Endoscopic papillectomy

\section{INTRODUCTION}

Acute renal cortical necrosis (RCN) accounts for approximately $2 \%$ of all cases of acute renal failure. RCN has a poor prognosis and usually progresses to chronic renal failure. ${ }^{1,2}$ RCN presumably occurs due to small renal blood vessel contraction, but this is unproven. RCN is primarily caused by complications occurring during pregnancy. ${ }^{3}$ However, the occurrence of RCN secondary to complications of pregnancy has decreased, while the incidences of non-pregnancy-related causes including viperine snake bite, hemolytic uremic syndrome, renal allograft rejection, acute gastroenteritis, acute pancreatitis, septicemia, and trauma have increased. ${ }^{1,3}$ Medications including non-steroidal anti-inflammatory drugs have

Received: January 17, 2017 Revised: May 18, 2017

Accepted: May 29, 2017

Correspondence: Seung Goun Hong

Department of Internal Medicine, SAM Anyang Hospital, 9 Samdeok-ro, Manan-gu, Anyang 14030, Korea

Tel: +82-31-467-9114, Fax: +82-31-449-0151, E-mail: permi@naver.com

(cc) This is an Open Access article distributed under the terms of the Creative Commons Attribution Non-Commercial License (http://creativecommons.org/ licenses/by-nc/3.0) which permits unrestricted non-commercial use, distribution, and reproduction in any medium, provided the original work is properly cited. rarely been reported to cause $\mathrm{RCN}^{3,4}$

Here we report a case in which acute RCN developed after tranexamic acid administration to treat gastrointestinal bleeding after an endoscopic papillectomy for an ampullary adenoma.

\section{CASE REPORT}

An 82-year-old woman with no significant medical history presented with a 2-month history of abdominal pain and nausea. Vital signs were as follows: blood pressure, 130/80 mm Hg; heart rate, 72 beats/minute (bpm); respiratory rate, 20/minute; and body temperature, $36.4^{\circ} \mathrm{C}$. She appeared chronically ill and the findings of a physical examination of the thorax, heart, and abdomen were unremarkable. Laboratory studies revealed a white blood cell (WBC) count of $8,110 / \mu \mathrm{L}$ (neutrophils, 78.5\%; and lymphocytes, $17.5 \%$ ) and a hemoglobin ( $\mathrm{Hb})$ of $11.5 \mathrm{~g} / \mathrm{dL}$. Serum biochemical values were as follows: blood urea nitrogen (BUN), $12 \mathrm{mg} / \mathrm{dL}$; serum creatinine (Cr), 0.8 $\mathrm{mg} / \mathrm{dL}$; aspartate aminotransferase/alanine aminotransferase, 21/14 U/L; albumin, $4.0 \mathrm{~g} / \mathrm{dL}$; total bilirubin, $0.6 \mathrm{mg} / \mathrm{dL}$; and amylase 2,460 U/L. Electrolyte levels were as follows: Na, 139 

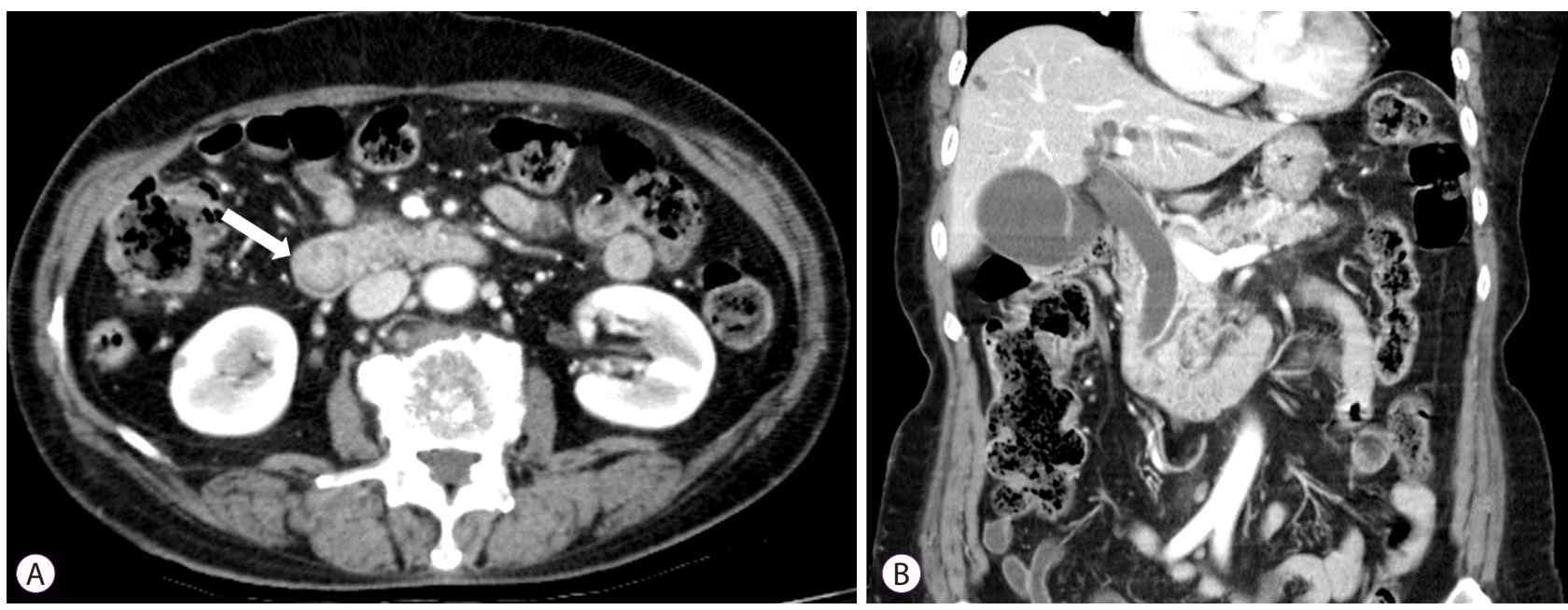

Fig. 1. Abdominal computed tomography scan revealing a 2-cm round mass (white arrow in A) near the ampulla of Vater, with mild to moderate dilation of the intrahepatic and common bile ducts as well as the pancreatic ducts (B). Both kidneys were normal in size without apparent abnormalities.

$\mathrm{mmol} / \mathrm{L} ; \mathrm{K}, 4.0 \mathrm{mmol} / \mathrm{L}$; and $\mathrm{Cl}, 102 \mathrm{mmol} / \mathrm{L}$. Carbohydrate antigen 19-9 (CA19-9) levels were $37.0 \mathrm{U} / \mathrm{mL}$.

An abdominal computed tomography (CT) scan revealed a 2-cm mass near the ampulla of Vater with mild dilation of the intrahepatic and common bile ducts as well as the pancreatic ducts (Fig. 1). There was no evidence of invasion of other organs. Both kidneys were normal sized without apparent abnormalities.

Endoscopic retrograde cholangiopancreatography with endoscopic ultrasound (EUS) revealed a 2 -cm adenoma-like protruding lesion in the ampulla of Vater. EUS showed a well-defined isoechoic homogeneous mass without bile or pancreatic duct invasion (Fig. 2A-C). The mass was resected using a snare and a plastic stent was inserted into the bile duct; insertion of a plastic stent into the pancreatic duct failed. No specific complications, including severe bleeding or perforation, were observed (Fig. 2D). Six hours post-procedure, the patient vomited $50 \mathrm{~mL}$ of blood and complained of abdominal pain. A second episode of hematemesis ( $<30 \mathrm{cc}$ ) occurred approximately 5 hours later. Immediately after the second episode, treatment with $1 \mathrm{~g}$ of tranexamic acid and $2 \mathrm{KU}$ of hemocoagulase administered three times per day along with ceftriaxone ( $2 \mathrm{~g}$ intravenous) and a proton pump inhibitor for suspected bleeding at the resection site was initiated. Her vital signs were unremarkable. The WBC count was $7,090 / \mu \mathrm{L}, \mathrm{Hb}$ was $10.1 \mathrm{~g} / \mathrm{dL}$, BUN was $14 \mathrm{mg} / \mathrm{dL}$, Cr was $0.8 \mathrm{mg} / \mathrm{dL}$, and amylase/lipase was 69/54 U/L. After the initial two episodes of hematemesis, no further hematemesis, melena, or hematochezia was observed and the patient's vital signs remained stable. $\mathrm{Her} \mathrm{Hb}$ level remained $>10 \mathrm{~g} / \mathrm{dL}$. Four days after the papillectomy, the patient's daily urine volume abruptly decreased to $<100 \mathrm{cc}$ and she complained of dyspnea. Her blood pressure was 103/86 mm Hg, heart rate was $134 \mathrm{bpm}$, respiratory rate was $30 /$ minute, and body temperature was $37.0^{\circ} \mathrm{C}$. Laboratory studies were as follows: $\mathrm{Hb}, 9.1 \mathrm{~g} / \mathrm{dL}$; amylase/lipase, 415/418 U/L; BUN/Cr, 73/3.9 mg/dL; and brain natriuretic peptide, $5,000 \mathrm{pg} / \mathrm{mL}$. Venous blood gas analysis revealed the following: $\mathrm{pH}, 6.92: \mathrm{pCO}_{2}, 37 \mathrm{~mm} \mathrm{Hg}$; and $\mathrm{HCO}_{3}, 7.6 \mathrm{mmol} /$ L. Pulmonary edema was observed on a chest radiograph and continuous renal replacement therapy (CRRT) was performed to treat metabolic acidosis and pulmonary edema caused by acute renal failure. Although there were no signs of bleeding at that time, 2 units of packed red blood cells were transfused.

Approximately 10 hours after the CRRT was initiated, the patient was noted to have melena mixed with a small amount of hematochezia. Melena continued to occur 2-3 times/day with a total daily volume of $200-400 \mathrm{cc}$. Her vital signs were relatively stable: blood pressure, 120-175/65-100 mm Hg; heart rate, $90-120 \mathrm{bpm}$; respiratory rate, $20-25 /$ minute; and body temperature, $36.0^{\circ} \mathrm{C}-37.8^{\circ} \mathrm{C}$. The daily serum $\mathrm{Hb}$ level was $7.1-9.5 \mathrm{~g} / \mathrm{dL}$ and the tranexamic acid with hemocoagulase were continued. The patient received a total of $13.5 \mathrm{~g}$ of tranexamic acid and $36 \mathrm{KU}$ of hemocoagulase and received three additional units of packed red blood cells until the sixth day post-procedure, when no further signs of bleeding were observed. However, her daily urine output was nearly zero despite the CRRT. A contrast-enhanced abdominal CT scan was performed on day 12 to assess the cause of her anuric acute renal failure and showed normal-sized kidneys with enhancement of the renal medulla but not of the renal cortex, a finding consistent with acute RCN (Fig. 3). Histopathology revealed a villotubular high-grade adenoma with clear resection margins. Conventional hemodialysis was continued, but renal function did not improve and the oliguria persisted post-discharge. Presently, 6 months after the endoscopic procedure, she continues to undergo hemodialysis. Her most recent 

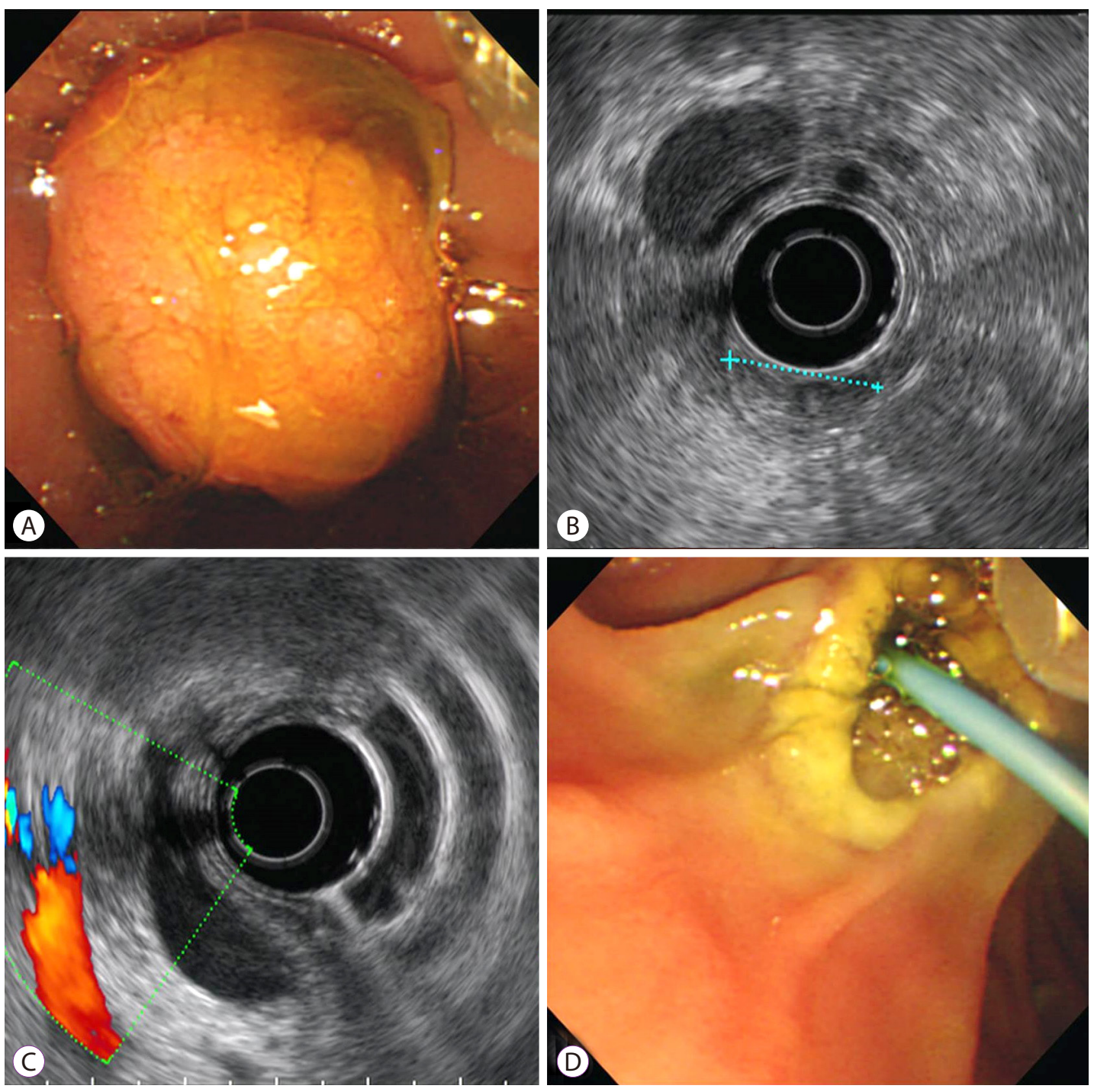

Fig. 2. A 2-cm adenoma-like protruding lesion was found in the ampulla of Vater on endoscopy (A) and endoscopic ultrasound showed an approximately 17-mm well-defined isoechoic homogeneous mass without bile duct or pancreatic duct invasion. A dilated common bile duct is visible (B, C). After mass resection using a snare and the insertion of a biliary plastic stent, no specific complications, such as severe bleeding or perforation, were observed (D).
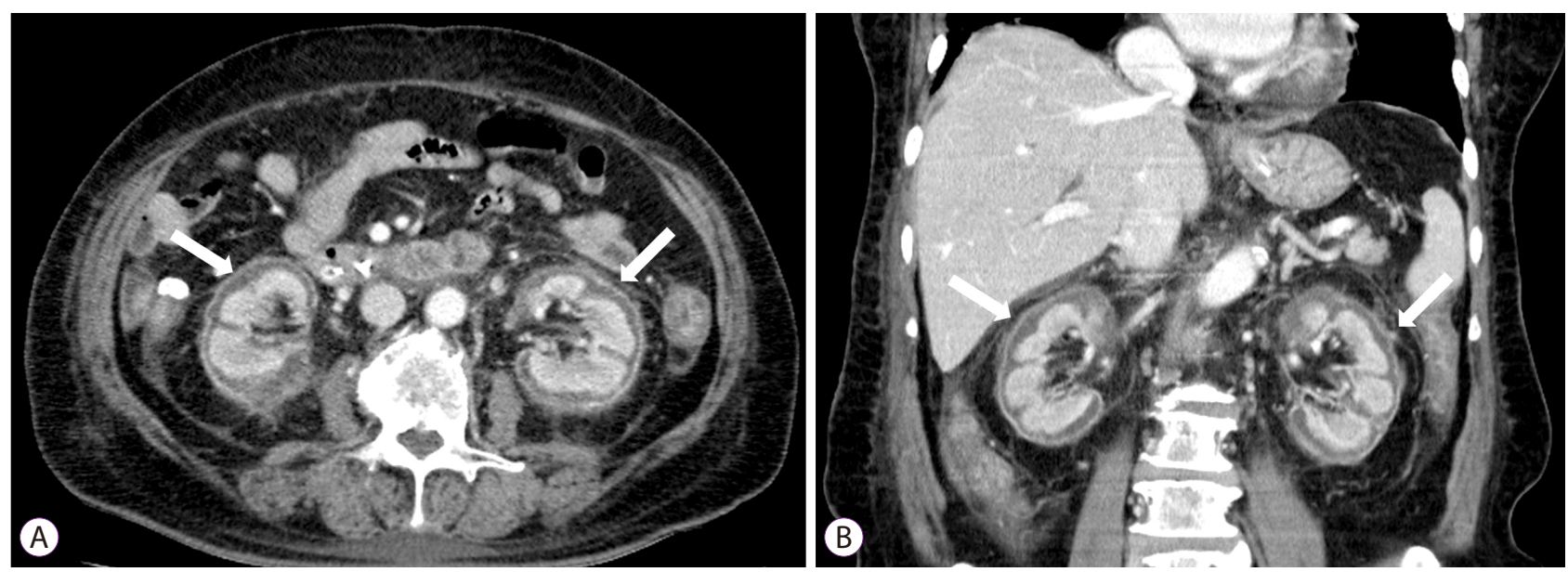

Fig. 3. Follow-up abdominal contrast-enhanced computed tomography scan revealing normal-sized kidneys with enhancement of the renal medulla but not the renal cortex (white arrows) consistent with acute renal cortical necrosis (A, B). 
BUN/Cr was 22/6.1 mg/dL.

\section{DISCUSSION}

Tranexamic acid infusion is a medical treatment for non-variceal upper gastrointestinal bleeding that decreases the need for surgical intervention and mortality. However, tranexamic acid alone is less frequently used than proton pump inhibitors or $\mathrm{H}_{2}$ receptor antagonists in these patients. ${ }^{5}$ Tranexamic acid reversibly binds plasminogen during thrombogenesis, interferes with the binding of plasminogen and fibrin, inhibits fibrinolysis, and thus exerts antifibrinolytic effects, promoting thrombus formation and hemostasis. Its side effects include mostly minor gastrointestinal symptoms (e.g., nausea, vomiting, abdominal pain, and diarrhea) and rarely severe complications related to thrombogenesis, such as pulmonary embolism, myocardial infarction, stroke, deep vein thrombosis, and RCN. ${ }^{6,7}$ To our knowledge, four cases of tranexamic acid-induced RCN have been reported to date, and ours is the first report of acute RCN in which tranexamic acid was administered for bleeding control after endoscopic papillectomy of an ampullary adenoma. ${ }^{8-11}$

In our patient, the volume of the first two episodes of hematemesis was relatively small, and there was no significant decrease in $\mathrm{Hb}$ level. Our patient's vital signs were stable before the development of anuric acute renal failure and dyspnea. Repeat endoscopy was considered because of the patient's additional bleeding events after CRRT but was not performed because the patient and her family preferred medical treatment alone after a thorough explanation of the risks and benefits of second-look endoscopy for hemostasis of the post-papillectomy bleeding. Fortunately, her bleeding stopped approximately 2 days after CRRT was initiated and 6 days after the endoscopic papillectomy.

Bleeding after endoscopic papillectomy has been reported to occur in $2 \%-16 \%$ of patients, and most episodes can be controlled with conservative management with medical treatment and endoscopic hemostasis. ${ }^{12}$ In our patient, the bleeding was successfully controlled by medical treatment and transfusions, but serious acute RCN occurred possibly because of renal artery vasoconstriction caused by tranexamic acid infusion. Hemocoagulase, which causes platelet aggregation at the bleeding site, accelerates fibrin hydrolysis and promotes thrombus formation. It is widely used to manage bleeding in various clinical settings, but no serious complications have been reported. ${ }^{13}$ Therefore, RCN was not likely to develop secondary to the administration of hemocoagulase, although the additive thrombotic effect of hemocoagulase may have acted synergistically with tranexamic acid to cause renal artery vasoconstriction.

Although the pathophysiology of RCN is poorly understood, significantly diminished renal arterial perfusion and the release of toxins results in the final common pathway of ischemic necrosis of the renal cortex. Endothelial injury, caused by the potent vasoconstrictor endothelin-1, has been implicated in the pathophysiology of RCN. Vascular endothelial injury can occur through a direct mechanism, as in hemolytic uremic syndrome, eclampsia, and snake bite, or indirectly as in sepsis, pancreatitis, and intravascular hemolysis. The diagnosis of RCN often requires a kidney biopsy, and the histology typically shows tubular cell necrosis with leukocyte infiltration. The glomeruli may be necrotic, and arteriolar thrombosis can be seen with medullary preservation and a thin rim of the subcapsular area. However, invasive biopsy has recently been replaced by non-invasive methods. Hallmark thin cortical lines caused by calcification can be observed on plain film, and a hypoechoic circumferential band in the renal subcapsular area is a characteristic finding on sonography. Contrast-enhanced CT scans are the most sensitive imaging modality, showing bilateral enhancement of the renal medulla and renal subcapsular area with no enhancement of the renal cortex. Renal scans are also useful if contrast-enhanced CT imaging is not available. . $^{1,2,14,15}$

Acute RCN can be treated with hemodialysis, as in other forms of acute renal failure. However, RCN has a poor prognosis and usually progresses to chronic renal failure. The mortality rate of RCN was $55 \%-86 \%$ prior to 1980 but decreased to $36 \%$ after 1980 with improvements in hemodialysis. Onethird of RCN patients still require continued hemodialysis. ${ }^{3}$ As shown in this rare case of tranexamic acid-induced acute RCN during gastrointestinal bleeding control, clinicians should be aware of the possibility of this serious complication.

\section{Conflicts of Interest}

The authors have no financial conflicts of interest.

\section{REFERENCES}

1. Prakash J, Singh VP. Changing picture of renal cortical necrosis in acute kidney injury in developing country. World J Nephrol 2015;4:480-486.

2. Sahay M, Swarnalata, Swain M, Padua M. Renal cortical necrosis in tropics. Saudi J Kidney Dis Transpl 2013;24:725-730.

3. Kim HJ. Bilateral renal cortical necrosis with the changes in clinical features over the past 15 years (1980-1995). J Korean Med Sci 1995;10:132141.

4. Aksoy S, Hocaoglu E, Karahasanoglu A, Igus B, Acay MB, Inci E. Bisphosphonate-induced bilateral acute renal cortical necrosis. Radiol Case Rep 2015;10:992.

5. British Society of Gastroenterology Endoscopy Committee. Non-variceal upper gastrointestinal haemorrhage: guidelines. Gut 2002;51 Suppl 4:iv1-iv6.

6. Gluud LL, Klingenberg SL, Langholz E. Tranexamic acid for upper gas- 
trointestinal bleeding. Cochrane Database Syst Rev 2012;1:CD006640.

7. Mannucci PM. Hemostatic drugs. N Engl J Med 1998;339:245-253.

8. Koo JR, Lee YK, Kim YS, Cho WY, Kim HK, Won NH. Acute renal cortical necrosis caused by an antifibrinolytic drug (tranexamic acid). Nephrol Dial Transplant 1999;14:750-752.

9. Levin MD, Betjes MG, V d Kwast TH, Wenberg BL, Leebeek FW. Acute renal cortex necrosis caused by arterial thrombosis during treatment for acute promyelocytic leukemia. Haematologica 2003;88:ECR21.

10. Odabaş AR, Cetinkaya R, Selçuk Y, Kaya H, Coşkun U. Tranexamic-acid-induced acute renal cortical necrosis in a patient with haemophilia A. Nephrol Dial Transplant 2001;16:189-190.

11. Park JH, Kang MK, Na WT, et al. A case of bilateral acute renal cortical necrosis complicated by tranexamic acid administration. Korean J Med
2011;80:723-728.

12. De Palma GD. Endoscopic papillectomy: indications, techniques, and results. World J Gastroenterol 2014;20:1537-1543.

13. Wang T, Wang DN, Liu WT, et al. Hemostatic effect of topical hemocoagulase spray in digestive endoscopy. World J Gastroenterol 2016;22:5831-5836.

14. Kim HJ, Cho OK. CT scan as an important diagnostic tool in the initial phase of diffuse bilateral renal cortical necrosis. Clin Nephrol 1996;45:125-130.

15. Lantsberg S, Rachinsky I, Lupu L, Tovbin D, Hertzanu Y. Unilateral acute renal cortical necrosis: correlative imaging. Clin Nucl Med 2000;25:184-186. 